\title{
Percepção das mães em relação ao desenvolvimento neuropsicomotor de seus filhos com doenças hepáticas: um estudo qualitativo
}

\author{
Mothers' perception of the neuropsychomotor \\ development of their children liver diseases: \\ a qualitative study
}

\author{
Juliana Costa Santos ${ }^{1}$ (i) \\ Nilo Manoel Pereira Vieira Barreto 2 (1) \\ Sumaia Midlej Pimantel Sá4 (i) \\ Hortência Souza Santana 3 (1) \\ Luciana Rodrigues Silva ${ }^{5}$
}

${ }^{1}$ Autora para correspondência. Universidade Federal da Bahia (Salvador). Bahia, Brasil. julicostasantos@hotmail.com

2,5 Universidade Federal da Bahia (Salvador). Bahia, Brasil. nilomanoel@gmail.com, lupe.ssa@uol.com.br ${ }^{3}$ Escola Bahiana de Medicina e Saúde Publica (Salvador). Bahia, Brasil. hortenciasantana17.1@bahiana.edu.br ${ }^{4}$ Universidade do Estado da Bahia (Salvador). Bahia, Brasil. sumaia.midlej@gmail.com

\begin{abstract}
RESUMO | INTRODUção: Muito se sabe sobre o quanto as doenças hepáticas impactam no desenvolvimento cognitivo, motor e social infantil. Entretanto, saber o que as mães pensam sobre o desenvolvimento de seus filhos e os aspectos que elas consideram mais importantes constituem temas pouco explorados. OBJETIVO: Investigar as percepções das mães de crianças hepatopatas acerca do desenvolvimento de seus fiIhos. MÉTODO: Trata-se de uma pesquisa qualitativa, descritivoexploratória, realizada com 12 mães de crianças (até 6 anos de idade) com hepatopatias crônicas, acompanhadas num centro de referência da Bahia. Realizou-se entrevista narrativa para coleta dos dados, que foram posteriormente transcritas e processadas conforme a análise de conteúdo temático categorial de Bardin. Foi comparado a percepção das mães acerca do desenvolvimento de seus filhos com os resultados obtidos na avaliação com o Teste de triagem de Denver II (TTDII) de suas crianças. RESULTADOS: Há concordância na maioria dos resultados da avaliação para suspeita ou risco do desenvolvimento neuropsicomotor, demonstrando que essas mães conseguem identificar comportamentos típicos e atípicos. As falas maternas são de superproteção e amenização do processo do adoecimento, bem como autoconfiança e superioridade como uma atitude de defesa, na tentativa de escamotear o sentimento de fragilidade frente a doença. CONCLUSÕES: A percepção das mães acerca do desenvolvimento de seus filhos foi convergente com os resultados do TTDII. As mães e as crianças precisam de acompanhamento multiprofissional para melhor manejo terapêutico e neurodesenvolvimento, bem como suporte familiar.
\end{abstract}

PALAVRAS-CHAVE: Doença Hepática Terminal. Deficiências do Desenvolvimento. Percepção
ABSTRACT | INTRODUCTION: Much is known about the impact of liver diseases on children's cognitive, motor, and social development. However, knowing what mothers think about their children's development and the aspects they consider most important are issues that have been little explored. OBJECTIVE: To investigate the perceptions of mothers of hepatopathy children about the development of their children. METHOD: This is a qualitative, descriptive-exploratory research conducted with 12 mothers of children (up to 6 years of age) with chronic liver diseases, followed by a reference center in Bahia, Brazil. A narrative interview was conducted to collect the data, later transcribed and processed according to Bardin's analysis of the category thematic content. The perception of mothers about the development of their children was compared with the results obtained in the evaluation with the Denver II screening test (TTDII) of their children. RESULTS: There is an agreement in most evaluation results for suspicion or risk of neuropsychomotor development, demonstrating that these mothers can identify typical and atypical behaviors. The maternal statements are of overprotection and softening of the disease process and self-confidence and superiority as an attitude of defense in an attempt to conceal the feeling of fragility in the face of the disease. CONCLUSIONS: The mothers' perception of their children's development was in line with the TDDII results. Mothers and children need multi-professional follow-up for better therapeutic management and neurodevelopment, as well as family support.

KEYWORDS: End Stage Liver Disease. Developmental Disabilities. Perception. 


\section{Introdução}

A doença crônica infantil é conhecida por ter o curso demorado ou indefinido. Algumas podem apresentar períodos de remissão e exacerbação de sintomas, necessitando de cuidados contínuos ${ }^{1}$, podendo cursar com internações, tratamentos invasivos e exaustivos, além da possibilidade de trazer prejuízos físicos e mentais para as crianças e impacto familiar. ${ }^{2}$ Com o diagnóstico de uma doença hepática crônica não é diferente, pois ela também requer regime de tratamento contínuo, incluindo uso ininterrupto de medicações, visitas periódicas aos médicos e realização de exames por toda a vida. $\mathrm{A}$ doença hepática caracteriza-se por lesão infamatória com aspecto fibrótico do órgão, com origem e níveis de necrose e inflamação hepatocelular variados, o que pode progredir até a cirrose. ${ }^{4}$ Suas diversas manifestações clínicas podem prejudicar o desenvolvimento e o crescimento na tenra idade.

O rápido neurodesenvolvimento, na primeira infância, faz com que qualquer injúria nessa fase possa gerar danos irreversíveis ao cérebro. Portanto, uma criança cujo fígado seja disfuncional pode se apresentar com alterações no seu desenvolvimento, pela liberação de substância neurotóxica. ${ }^{5}$ Muito se sabe sobre o quanto as doenças hepáticas impactam no desenvolvimento cognitivo, motor e social infantil. $\underline{6}$ Entretanto, saber o que as mães pensam sobre o desenvolvimento de seus filhos e os aspectos que elas consideram mais importantes constituem temas pouco explorados. Anton e Piccinini ${ }^{10}$, ao investigarem o impacto da doença crônica e do transplante hepático no desenvolvimento emocional de crianças hepatopatas, criaram uma categoria sobre desenvolvimento. As mães relataram que o desenvolvimento motor, antes do transplante, era atrasado, e, após essa cirurgia, observaram melhora. As mães descreveram, ainda, que seus filhos eram bem comunicativos e inteligentes, porém a maioria ainda se apresentava dependente em alguns aspectos, tais como nos hábitos pessoais de higiene e no desejo

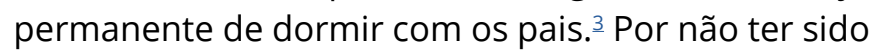
um estudo com objetivo principal focado no neurodesenvolvimento das crianças hepatopatas, buscou-se na literatura outros estudos que relacionassem escalas de desenvolvimento neuropsicomotor com o relato de mães no contexto destas crianças, sem a obtenção de resultados. Acredita-se que, muitas vezes, as mães subestimam e ou superprotegem suas proles, principalmente em uma situação de adoecimento, ou mesmo apresentam atitudes opostas de otimização das habilidades das crianças. Nessa perspectiva, o objetivo deste estudo foi investigar as percepções das mães de crianças hepatopatas sobre desenvolvimento neuropsicomotor de seus filhos. atendidas em centro de referência no estado da Bahia.

\section{Métodos}

Trata-se de um estudo qualitativo, descritivo e exploratório, no qual doze mães e seus filhos com idades entre seis meses a seis anos, de ambos os sexos, foram selecionadas. Crianças com hepatopatias crônicas e que fossem atendidas no Centro Pediátrico Prof. Hosannah Oliveira - Complexo Hospitalar Universitário Professor Edgard Santos da Universidade Federal da Bahia (HUPES-UFBA), no ambulatório de gastroenterologia e hepatologia pediátrica, em Salvador, Bahia.

Foram excluídas as mães cujas crianças apresentavam distúrbios neurológicos e psiquiátricos ou outras doenças crônicas, que apresentavam alguma síndrome genética, crianças surdas ou cegas, com trauma cranioencefálico, hemodinamicamente instáveis, e aquelas cujos pais ou responsáveis não assinaram o Termo de Consentimento Livre e Esclarecido (TCLE). 
A coleta de dados foi realizada no período de novembro de 2019 a março de 2020 e as entrevistas e avaliação do desenvolvimento neuropsicomotor foram realizadas no mesmo dia de consulta com médico hepatologista em um consultório específico para pesquisa. No primeiro contato com as mães, realizou-se a explicação do trabalho, com a leitura do TCLE, que, em seguida, foi aceito e assinado. Logo após, aconteceu a aplicação do questionário sociodemográfico e a realização da entrevista narrativa, com a seguinte pergunta disparadora: "O que a senhora acha do desenvolvimento do seu filho ou sua filha?" As entrevistas foram gravadas em um iPhone 7s para posterior transcrição de dados.

As entrevistas foram analisadas e transcritas pelos autores, quando foi possível estabelecer as relações e os pontos comuns às falas das entrevistadas baseando-se na análise do conteúdo proposta por Bardin. ${ }^{7}$ As categorias que emergiram das narrativas das mães foram: "Ele é normal": percepção das mães acerca do desenvolvimento de seus filhos" e "É... acho que é normal": percepção das mães a partir da doença hepática". As mães entrevistadas foram denominadas apenas como mãe 1 (M1), mãe 2 (M2), mãe 3 (M3), mãe 4 (M4), e assim sucessivamente, preservando-se o sigilo quanto à sua identidade. As transcrições das falas seguem literalmente o modo como cada mãe fez o seu relato, não havendo adequação ou correção gramatical.

Após a entrevista com as mães, a avaliação do Desenvolvimento Neuropsicomotor (DNPM) foi realizada com os filhos. O instrumento de avaliação do desenvolvimento utilizado foi o Teste de Triagem Denver II (TTDII), que foi aplicado por uma fisioterapeuta habilitada. Esse instrumento é classificado dicotomicamente em "suspeito ou risco para o desenvolvimento" ou "normal". Contém 125 itens, apresentados em quatro domínios: pessoal-social, motor fino-adaptativo, linguagem e motor grosso. ${ }^{\circ}$ A avaliação não é invasiva, é de fácil aplicação, muito parecida com as brincadeiras cotidianas das crianças, o tempo de aplicação dura em média 30 minutos.
$\mathrm{Na}$ aplicação do teste, calcula-se a idade da criança e, em seguida, traça-se uma linha vertical na folha específica do instrumento, quando, então, se aplicam os procedimentos referentes a essa faixa etária em todos os domínios. A criança é classificada como normal quando apresenta nenhum "atraso" ou, no máximo, uma "cautela" em todo teste; caso apresente uma ou mais "cautela" e (ou) um ou mais "atraso", ela é classificada como suspeita, com risco para alteração do DNPM. Todas as etapas (entrevistas com as mães e avaliação do DNPM das crianças) foram realizadas em um tempo médio de 1 hora.

O estudo obedeceu às recomendações da Resolução CNS 466 de 12 de dezembro de 2012 para o desenvolvimento de pesquisa com seres humanos. Todos os indivíduos que concordaram em participar do estudo assinaram o TCLE. Este projeto foi aprovado pelo Comitê de Ética em Pesquisa do Instituto de Ciências da Saúde, ICS/UFBA sob o parecer de $n^{\circ} 3.695 .203$ (CAAE 09357019.2.0000.5662).

\section{Resultados e discussão}

Participaram do estudo 12 mães de crianças com doenças hepáticas crônicas. As idades das mães variaram de 22 a 45 anos, e as idades das crianças de nove meses a seis anos. A renda de onze famílias foi de até dois salários mínimos (classes C2 e D-E pela APEB). A maioria das mães tinha ensino médio completo como maior escolaridade na amostra, eram solteiras e residentes no interior da Bahia. Oito crianças eram do sexo masculino e sete tinham irmãos. Em relação às hepatopatias, oito tinham fígado preservado (nativo) e quatro realizaram transplante hepático. Sete crianças apresentaram suspeita ou risco para o desenvolvimento neuropsicomotor segundo o TTDII. Houve cinco discordâncias entre o resultado do TTDII e a percepção das mães, (Quadro 1). 
Quadro 1. Caracterização das mães e das crianças de seis meses a seis anos com hepatopatias crônicas

\begin{tabular}{|c|c|c|c|c|c|c|c|c|}
\hline Nome & $\begin{array}{c}\text { Gênero } \\
\text { Filhos }\end{array}$ & Cidade & Classificação APEB 1 & TTDII & Relato das Mães & Percepção Mães x TTDII & Diagnóstico & Irmãos \\
\hline $\begin{array}{c}\text { Criança } \\
\text { (6A) } \\
\text { Mãe } 1\end{array}$ & Masculino & Interior & $\begin{array}{c}C 2 \\
(1.691 \$ R)\end{array}$ & Normal & Normal & Concordância & Fígado nativo & 1 \\
\hline $\begin{array}{c}\text { Criança } \\
(5 A) \\
\text { Mãe } 2 \\
\end{array}$ & Masculino & Interior & $\begin{array}{c}C 1 \\
(2.965 \$ R)\end{array}$ & Suspeita ou risco & Suspeita & Concordância & Fígado nativo & 2 \\
\hline $\begin{array}{c}\text { Criança } \\
(4 A) \\
\text { Mãe } 3\end{array}$ & Feminino & Interior & $\begin{array}{c}C 2 \\
(1.691 \$ R)\end{array}$ & Normal & Normal & Concordância & Fígado nativo & 0 \\
\hline $\begin{array}{c}\text { Criança } \\
(4 A) \\
\text { Mãe } 4\end{array}$ & Masculino & Salvador & $\begin{array}{c}\mathrm{D}-\mathrm{E} \\
(708 \$ \mathrm{R})\end{array}$ & Normal & Normal & Concordância & Fígado nativo & 1 \\
\hline $\begin{array}{c}\text { Criança } \\
(4 A) \\
\text { Mãe } 5\end{array}$ & Feminino & Interior & $\begin{array}{c}C 2 \\
(1.691 \$ R)\end{array}$ & Suspeita ou risco & Suspeita & Concordância & Fígado nativo & 2 \\
\hline $\begin{array}{c}\text { Criança } \\
(5 A) \\
\text { Mãe } 6 \\
\end{array}$ & Masculino & Interior & $\begin{array}{c}\mathrm{D}-\mathrm{E} \\
(708 \$ \mathrm{R})\end{array}$ & Suspeita ou risco & Normal & Discordância & Fígado nativo & 1 \\
\hline $\begin{array}{c}\text { Criança } \\
(9 M) \\
\text { Mãe } 7\end{array}$ & Masculino & Outros & $\begin{array}{c}\mathrm{D}-\mathrm{E} \\
(708 \$ \mathrm{R})\end{array}$ & Suspeita ou risco & Normal & Discordância & Fígado nativo & 1 \\
\hline $\begin{array}{c}\text { Criança } \\
(5 A) \\
\text { Mãe 8 }\end{array}$ & Masculino & Salvador & $\begin{array}{c}B 2 \\
(5.363 \$ R)\end{array}$ & Suspeita ou risco & Normal & Discordância & Fígado nativo & 0 \\
\hline $\begin{array}{c}\text { Criança } \\
(2 A) \\
\text { Mãe } 9\end{array}$ & Feminino & Interior & $\begin{array}{c}C 2 \\
(1.691 \$ R)\end{array}$ & Suspeita ou risco & Normal & Discordância & Transplante hepático & 0 \\
\hline $\begin{array}{c}\text { Criança } \\
\text { (3A) } \\
\text { Mãe } 10\end{array}$ & Feminino & Interior & $\begin{array}{c}C 2 \\
(1.691 \$ R)\end{array}$ & Suspeita ou risco & Normal & Discordância & Transplante hepático & 0 \\
\hline $\begin{array}{c}\text { Criança } \\
(16 \mathrm{M}) \\
\text { Mãe } 11\end{array}$ & Masculino & Salvador & $\begin{array}{c}C 2 \\
(1.691 \$ R)\end{array}$ & Normal & Normal & Concordância & Transplante hepático & 0 \\
\hline $\begin{array}{l}\text { Criança } \\
(4 A) \\
\text { Mãe } 12\end{array}$ & Masculino & Interior & $\begin{array}{c}\mathrm{D}-\mathrm{E} \\
(708 \$ \mathrm{R})\end{array}$ & Normal & Normal & Concordância & Transplante hepático & 1 \\
\hline
\end{tabular}

1 Salário mínimo em reais em 2020 de $\mathrm{R} \$ 1.045,00$. A= anos; $M=$ meses 


\section{"Ele é normal": percepção das mães acerca do desenvolvimento de seus filhos}

Desenvolvimento é capacidade de que a criança precisa para desempenhar funções e atividades cada vez mais complexas, tratando-se de processos dinâmicos com alterações físicas, sociais, emocionais, de linguagem e cognitivas.. No nosso estudo, dez mães relataram que o desenvolvimento de suas crianças era normal. Observou-se que a Mãe 1 e a Mãe 9 supervalorizaram o desenvolvimento de seus filhos:

"É de acordo com a idade, bem avançado às vezes, no caso assim pra idade dele." (M1)

"Ah... o desenvolvimento dela foi bem ótimo, assim... Eu não achava que ia ser tão rápido, assim pelo fato dela ser transplantada." (M9)

Sabe-se do impacto que uma doença crônica pode trazer ao seio familiar. Mudança de rotina diária, com visitas constantes ao médico, administração de medicações, exames, internações hospitalares, redução da participação social em escolas e comunidades - tudo isso pode comprometer o desenvolvimento neuropsicomotor da criança em diversas esferas. ${ }^{10}$ Observou-se que o filho da Mãe 9 apresentou, segundo o TTDII, suspeita ou risco para DNPM, ou seja, houve discordância da percepção da mãe, que não enxergou alteração alguma em seu filho. Acredita-se que essa mãe atribuiu um bom desenvolvimento a seu filho pensando no aspecto biológico da doença fatal e a gravidade de realizar um procedimento complexo, como transplante hepático. Ela, de certa forma, observou melhoras no desenvolvimento dele após esse procedimento. Outra questão que pode ter influenciado na visão otimista da mãe seria o fato de ela ter o comparativo da criança pré e pós-transplante. A literatura indica melhora das habilidades neuromotoras após transplante hepático, embora essas crianças ainda permaneçam abaixo da média preconizada para a idade. ${ }^{11,12}$

As mães 6, 7, 8 e 10 também foram discordantes sobre a desenvolvimentos de seus filhos quando comparados ao TTDII:

"Normal, ele assim em termo da visão a gente, dá os objetos, ele acompanha o som quando o barulho tá muito alto, incomoda ele, ele já rola na cama, ele rola na cama já. Tem que ter toda atenção, eu acho que o desenvolvimento dele tá normal." (M7)
A mãe 7 demostrou acompanhar o desenvolvimento motor do filho que tinha menos de um ano de idade, pois o relato veio com informações sobre esse domínio. O TTDII dessa criança apresentou "cautela e atenção" nos domínios pessoal social e linguagem, o que demonstra a limitada visão da mãe acerca do desenvolvimento como um todo. Para Hanton et al., os pais tendem a negar a possibilidade de existirem aspectos negativos que a doença pode trazer para seus filhos, como uma tentativa de defesa. ${ }^{13}$ Logo, pode existir uma valorização dessa mãe da habilidade que a criança realiza bem, em detrimento de outras, que não estejam compatíveis com a idade da criança.

Duas mães (4 e 10) trouxeram, em seus discursos, uma associação do desenvolvimento com aspectos cognitivos:

"Às vezes, eu acho ele desatento em algumas coisas, mas, no mais, ele tem uma percepção muito boa. Desde muito cedo, ele sabia boca, nariz e tal. As cores ele aprendeu muito rápido. Então, assim em relação

a isso, eu acho que ele desenha direitinho. Quando ele tá com preguiça é quando ele risca tudo assim de qualquer jeito, mas agora há pouco ele estava com um papelzinho que..." (M4)

"Eu acho que ela tem um desenvolvimento compatível com a idade. No colégio, ela tá indo muito bem, ela escreve o nome completo. E a avaliação que a professora e que a escola me dá é que ela está desenvolvendo bem." (M10)

A escola e os consequentes aprendizados cognitivos e sociais podem funcionar como um marco para esses pais, pois é um ambiente de confrontamento com padrões mais rígidos de desempenho, onde as crianças serão expostas, fora do convívio familiar, as inevitáveis comparações com seus colegas..$^{14}$ Isso nos faz pensar que essas mães se sentem contempladas acerca do desenvolvimento quando seus filhos aprendem e demonstram aprendizado no dia a dia, assim como quando acompanham os colegas da escola.

Anton \& Augusto ${ }^{3}$, investigaram a percepção das mães sobre o desenvolvimento de crianças de quatro a oito anos, no contexto do transplante hepático. As mães relataram, nesta casuística, o impacto negativo da hepatopatia pré-transplante e a melhora, com o passar do tempo, e o sucesso do transplante no desenvolvimento físico e da comunicação social. ${ }^{3}$ 
Resultados semelhantes foram encontrados no nosso estudo, quando analisada a fala da mãe 11: "Atrasou por conta do transplante, depois do
transplante aí deslanchou" (M11)

Outra variável analisada no estudo acima foi o teste de fábulas, que revelou conflitos emocionais e prejuízo no desenvolvimento emocional maior do que o percebido pelas mães. Ou seja, houve discordâncias entre dados de um teste e a percepção dos pais. ${ }^{3}$ No presente estudo, no caso das crianças com suspeita ou risco para o DNPM pelo TTDII, 5/7 mães não perceberam alterações no desenvolvimento, revelando também uma discordância entre um teste e a percepção maternal. Por mais que não tenham sido maioria, essas famílias precisam ser assistidas, pois o fato dessas mães supervalorizarem o desenvolvimento de seus filhos pode trazer implicações, privando essas crianças de intervenções oportunas para minimizar os riscos ao desenvolvimento.

\section{“É... acho que é normal": percepção das mães a partir da doença hepática}

Crianças com doença hepática crônica apresentam atraso em diversas esferas do desenvolvimento neuropsicomotor. ${ }^{6,15} \mathrm{~A}$ doença de base, que compromete as funções múltiplas do fígado, muitas vezes provoca desnutrição (que gera perda de massa muscular e fraqueza), presença de ascite, internações recorrentes, e diminui, de modo claro, as oportunidades para a realização das experiências neuromotoras fundamentais para a obtenção de um bom desenvolvimento, prejudicando a capacidade funcional em casa e na escola. ${ }^{6}$ O relato da Mãe 5 traz conteúdos a respeito de como a hepatopatia influencia a execução de habilidades cotidianas de uma criança:

"Assim, normal, normal, não chega a ser né? Por conta da barriga dela ser muito grande, isso incomoda ela muito né? E, a vezes, atrapalha, mas até que ela faz tudo que uma criança normal faz: ela brinca, pula, às vezes se joga, cai de barriga no chão, e eu fico preocupada o tempo todo. Ela não para quieta e assim a questão dela sentir muitas dores, todo dia praticamente ela sente dor, "aí tá doendo", aí toma remedinho. Ultimamente, ela começou a sentir dores nas pernas. Todo santo dia agora ela reclama de dores nas pernas. Aí eu não sei se tem alguma coisa a ver, porque ela começou a sentir tem um mês. Então aí não sei... Mas tirando isso...". (M5)
Nessa fala, podemos observar que a mãe faz correlações entre o desenvolvimento da filha e a doença hepática, que limita a criança em suas brincadeiras, como se os atrasos no DNPM ficassem em segundo plano, segundo o ponto de vista desta mãe. Para essa mãe, o mais importante é o componente biológico e fatal da patologia. A Mãe 2 também apresentou incerteza em seu relato. Acredita-se que, naquelas crianças com maior gravidade e maiores manifestações da doença crônica, o desenvolvimento da motricidade, da linguagem, de habilidades sociais e cognitivas se apresentaram sem muita importância para suas cuidadoras. O que nos faz refletir que, para essas mães, a hepatopatia pode ter um desfecho fatal, enquanto que desvios no desenvolvimento das crianças não levariam à morte.

"Ele é aparentemente normal, ele é normal. Mas ele tem que ser observado por causa da glicemia, ele tem a queda de glicemia. Aí tem que tá alimentando direitinho, no horário certo, não pode faltar os horários, tudo certinho. Eu monitoro a glicemia 3 vezes no dia, 2 vezes quando necessário." (M2)

Os resultados do TTDII das crianças das mães 2 e 5 , que demonstraram suspeita ou risco para DNPM, concordaram com o relato das cuidadoras. Vários aspectos podem ter influenciado para que $58 \%$ da atual casuística tenha apresentado desvios no Denver II, como renda familiar baixa, pois quanto maior o status socioeconômico, maiores oportunidades essas crianças têm de serem estimuladas. Nossa casuística tinha renda familiar predominante de 2 salários mínimos. ${ }^{16}$ Quanto ao grau de instrução dos pais, a literatura aponta que quanto maior a educação dos pais (diploma universitário ou nível mais elevado), melhores escores cognitivos dos filhos. ${ }^{17}$ Sabe-se o quanto fatores de risco socioambientais podem influenciar na aquisição das habilidades do neurodesenvolvimento, e que as crianças precisam de um ambiente favorável para esse benefício, logo, quando se associam fatores de risco biológicos e socioeconômicos, os prejuízos para o desenvolvimento são evidentes.

Os dados oriundos desta pesquisa, de cunho qualitativo, têm baixo poder de generalização, por tratar de aspectos subjetivos da percepção de mães de crianças hepatopatas acerca do desenvolvimento dos seus filhos. A interpretação dos dados está relacionada à subjetividade do pesquisador, ou seja, a forma como este interpreta o que lhe foi dito, a partir da sua história, e dos entendimentos anteriores. 
Nossos dados podem ser utilizados para advertir a equipe multiprofissional que acompanha essas crianças e seu ciclo familiar sobre os possíveis desdobramentos no neurodesenvolvimento, para que não só os pacientes sejam melhores assistidos, mas também seus familiares. Programas de reabilitação com neuropsicólogos, fisioterapeutas, nutricionistas, além de outros profissionais, poderiam ser instituídos, já que com aumento da sobrevivência será mais comum encontrar pacientes com risco de se desenvolver em diversas esferas.

\section{Conclusões}

De forma geral, a percepção das mães acerca do desenvolvimento de seus filhos coincidiu com os resultados do TTDII, demonstrando que essas mães conseguem identificar comportamentos típicos e atípicos. A doença hepática crônica pode trazer consequências só físicas e emocionais, por se tratar de uma patologia que exige muitas modificações no âmbito familiar. Pudemos observar, também, falas maternas de superproteção e amenização do processo do adoecimento, assim como relatos de autoconfiança e superioridade. Tais comportamentos podem configurar uma atitude de defesa, na tentativa de escamotear o sentimento de fragilidade que a doença provoca.

Cabe salientar que o fato de a maioria das crianças deste estudo apresentar risco potencial de atraso no DNPM nos alerta para a necessidade da realização de novos testes para confirmação diagnóstica, visto que o TTDII é um instrumento de triagem. $O$ estudo evidencia que essas mães e suas crianças precisam de acompanhamento multiprofissional, para que a abordagem terapêutica seja conduzida sem ônus significativos ao desenvolvimento neuro-psicossocial das crianças e de suas famílias.

\section{Contribuições dos autores}

SantosJC, Barreto NMPV, Sá SMP, Santana HS e Silva LR participaram da concepção do projeto, coleta, análise e interpretação dos dados, redação do artigo e revisão crítica relevante do conteúdo intelectual.Todos os autores aprovaram a versão final.

\section{Conflitos de interesses}

Nenhum conflito financeiro, legal ou político envolvendo terceiros (governo, empresas e fundações privadas, etc.) foi declarado para nenhum aspecto do trabalho submetido (incluindo, mas não se limitando a subvenções e financiamentos, participação em conselho consultivo, desenho de estudo, preparação de manuscrito, análise estatística, etc.).

\section{Referências}

1. Nóbrega VM, Silva MEA, Fernandes LTB, Viera CS, Reichert APS, Collet N. Doença crônica na infância e adolescência: continuidade do cuidado na Rede de Atenção à Saúde. Rev esc enferm USP. 2017;51:e03226. http://dx.doi.org/10.1590/S1980220X2016042503226

2. Castro EK, Piccinini CA. A experiência de maternidade de mães de crianças com e sem doença crônica no segundo ano de vida. Estud Psicol. 2004;9(1):89-99. https://doi.org/10.1590/S1413294X2004000100011

3. Anton MC, Augusto C. O desenvolvimento emocional em crianças submetidas a transplante. Estud Psicol. 2011;16(1):39-47. https://doi.org/10.1590/S1413-294X2011000100006

4. Macedo AF, Azevedo RA, Zanin FAA, Duarte D. Manifestações bucais e sistêmicas em crianças com doença hepática crônica. RGO (Porto Alegre) [Internet]. 2007;55(4):403-6. Disponível em: https://pesquisa.bvsalud.org/portal/resource/pt/lil-510976

5. Parekh PJ, Balart LA. Ammonia and Its Role in the Pathogenesis of Hepatic Encephalopathy. Clin Liver Dis. 2015;19(3):529-37. https://doi.org/10.1016/j.cld.2015.05.002

6. Rodijk LH, Den Heijer AE, Hulscher JBF, Verkade HJ, De Kleine RHJ, Bruggink JLM. Neurodevelopmental outcomes in children with liver diseases: A systematic review. J Pediatr Gastroenterol Nutr. 2018;67(2):157-68. https://doi.org/10.1097/ MPG.0000000000001981

7. Bardin L. Análise de Conteúdo. Lisboa: Edições 70; 2007.

8. Sabatés AL. Denver II: Teste de triagem do desenvolvimento: Manual de treinamento. São Paulo: Hogrefe; 2017.

9. Fonseca E. Desenvolvimento Normal de 1 a 5 anos. Rev Pediatr SOPERJ [Internet]. 2011;12(suppl 1):4-8. Disponível em: http:// revistadepediatriasoperj.org. br/detalhe artigo.asp?id=551

10. Anton MC, Piccinini CA. The impact of pediatric liver transplantation on family relationsvO impacto do transplante hepático infantil na dinâmica familiar. Psicol Reflex Crit. 2010;23(2):187-97. https://doi.org/10.1590/S010279722010000200001 
11. Almaas R, Jensen $U$, Loennecken MC, Tveter AT, Sanengen $\mathrm{T}$, Scholz T, et al. Impaired motor competence in children with transplanted liver. J Pediatr Gastroenterol Nutr. 2015;60(6):723-8. https://doi.org/10.1097/MPG.0000000000000757

12. De-Paula EM, Porta G, Tannuri ACA, Tannuri U, Befi-Lopes DM. Language assessment of children with severe liver disease in a public service in Brazil. Clinics. 2017;72(6):351-7. https://doi. org/10.6061/clinics/2017(06)04

13. Hanton LB. Caring for children awaiting heart transplantation: psychosocial implications. Pediatr Nurs. 1998;24(3):214-8. Citado em: PMID: 9987419.

14. Galvão BAP, Bueno KMP, Rezende MB, Magalhães LC. Percepção materna do desempenho de crianças com transtorno do desenvolvimento da coordenação. Psicol em Estud. 2014;19(3):527-38. https://doi.org/10.1590/1413-73722039315
15. Ng VL, Sorensen LG, Alonso EM, Fredericks EM, Ye W, Moore $\mathrm{J}$, et al. Neurodevelopmental Outcome of Young Children with Biliary Atresia and Native Liver: Results from the ChiLDReN Study. J Pediatr. 2018;196:139-147.e3. https://doi.org/10.1016/j. jpeds.2017.12.048

16. Halpern R, Giugliani ERJ, Victora CG, Barros FC, Horta BL. Risk factors for suspicion of developmental delays at 12 months of age Fatores de risco para suspeita de atraso no desenvolvimento neuropsicomotor aos 12 meses de vida. Rev Chil pediatría. 2002;73(5):529-39. http://dx.doi.org/10.4067/S037041062002000500016

17. Squires JE, Ng VL, Hawthorne K, Henn LL, Sorensen LG, Fredericks EM, et al. Neurodevelopmental Outcomes in Preschool and School Aged Children with Biliary Atresia and Their Native Liver. J Pediatr Gastroenterol Nutr. 2020;70(1):79-86. https://doi. org/10.1097/mpg.0000000000002489 